\title{
Sprechen und Vortragen mit Gedichten - Ästhetische Kommunikation im Fremdsprachenunterricht
}

\author{
Anke Stöver-Blahak
}

„Ich habe es eher als ein Arbeitswerkzeug betrachtet als ein richtiges Gedicht."

Der Workshop - Der Workshop ist zweigeteilt: Im ersten Teil erhalten die TeilnehmerInnen eine kurze Einführung in die theoretischen Grundlagen (Konstruktivismus, Lernerautonomie, Sprecherziehung, Ästhetische Kommunikation) und bekommen einen Überblick über die Rahmenbedingungen, unter denen das vorgestellte Konzept in der Praxis umgesetzt wird: studienbegleitender Kurs Deutsch als Fremdsprache am Fachsprachenzentrum, für alle Fakultäten, alle Studienabschlüsse, 13 Wochen, 2 SWS, durchschnittlich 10 Kurs-TeilnehmerInnen, Raum mit Videokameras.

Im zweiten Teil werden in gestraffter Form alle Elemente des Sprechens und Vortragens mit den im Kurs eingesetzten Übungen durchgespielt. Es beginnt mit Übungen zum Auftreten, zur Körperhaltung, Atmung, zu Einzellauten und suprasegmentalen Phänomenen. Dann werden in Kleingruppen drei Gedichte erarbeitet und drei Videoaufnahmen gemacht. Diese werden, wie im Kurs, gemeinsam angesehen und besprochen. Zugrunde liegen Kriterien für einen guten Vortrag, wie sie von Studierenden im Kurs entwickelt wurden. Die Workshop-TeilnehmerInnen durchlaufen so zumindest einmal eine Feedbackschleife (innerer Monitor, Peer-Feedback, Videoaufzeichnungen) und reflektieren ihre eigene sowie die „Sprechleistung“ der anderen. Sie können erfahren, wie sich auf diesem Weg eine Sprechfassung entwickelt, die sowohl der vortragenden Person, als auch dem Gedicht und der Vortragssituation gerecht wird. Zwei Videobeispiele eines Kursteilnehmers vom Anfang und Ende des Kurses illustrieren einen möglichen Erfolg.

Gemeinsames Erleben - Durch das gemeinsame Erleben mit Kopf, Herz, Hand und Fuß in einem Workshop lässt sich das Konzept deutlich leichter vermitteln als durch die übliche Vortragsform. Allein und doch geschützt durch eine solidarische und unterstützende Gruppe können die WorkshopTeilnehmerInnen ebenso wie die Kurs-TeilnehmerInnen sich selbst in einer Vortragssituation erleben. Sie können über ihr eigenes Sprechen reflektieren, durch das Feedback der anderen und durch die Videoaufnahme neue Aspekte 
ihres Sprechens entdecken, vielleicht schon Gelerntes sichern oder bestätigen, Neues ausprobieren - sie erhalten einen Rahmen und einen Raum für Erfahrungen mit sich selbst. Aufgabe der Dozentin im Kurs und im Workshop ist es, diesen Raum zur Verfügung zu stellen und zu sichern. Darüber hinaus muss sie während des Workshops den Bezug zur Praxis herstellen - durch Erklärungen im ersten Teil, während des Workshops und durch eine Reflexionsrunde am Ende.

Durch die sprechende Erarbeitung einer Vortragsfassung eines Gedichts wird auch der „Werkzeugcharakter“ des Gedichts deutlich. Es „dient“ in der Tat als Hilfsmittel - inhaltslos kann man nicht Sprechen üben. Aber spätestens beim Nachdenken über Betonungen, Prosodie oder Pausen beginnt das Interpretieren. Das Gedicht behält seine eigene poetische Würde. Ziel ist eine Sprechfassung, die alle drei Aspekte umfasst: Vortragende/r, Gedicht und Vortragssituation.

Ziel des Workshops - Ziel des Workshops war es, KollegInnen dieses Konzept „erfahrbar" zu machen und sie zu ermutigen, es auch in ihren Kontexten anzuwenden bzw. ihre Studierenden auf diese Art zu üben aufmerksam zu machen. Sprechen und Vortragen sind Kompetenzen, die im akademischen Kontext einerseits unerlässlich und positiv formuliert entscheidend für den kommunikativen Erfolg sind. Vortragssituationen sind immer performativ und lassen sich nur begrenzt rein kognitiv erschließen. Insbesondere Fremdsprachenlernende stehen hier oft vor großen - ganz unterschiedlich gearteten - Hürden. Indem man ihnen Raum und Zeit zur Verfügung stellt, in einem geschützten Rahmen performativ an individuell gesetzten Zielen zu arbeiten, festigt man zum einen ihre Fremdsprachenkenntnisse - aber gleichzeitig stützt man das Selbstbewusstsein, vermittelt durch die Gedichte auch Kulturwissen und macht sie vertraut mit den vielen Aspekten von Performativität in ihrem Studienalltag.

An Hochschulen wird überwiegend kognitiv gearbeitet und vermittelt. Den wenigsten ist die Inszeniertheit des eigenen Auftretens oder des Auftretens anderer bewusst. Dieses Konzept kann dazu beitragen, die Teilnehmenden darauf aufmerksam zu machen - indem sie es mit allen Aspekten: Kognition, Emotionalität und Körper erfahren.

Fremdsprachenunterricht an Universitäten hat zunächst eine "dienende“ Funktion. Die Studierenden lernen die Sprache, um ihr Studium besser oder überhaupt bewältigen zu können. Der Unterricht muss so konzipiert sein, dass sie einen Nutzen für sich selbst erkennen - idealerweise sollte dieser Nutzen aber auch den Dozierenden, Professoren und Professorinnen deutlich sein, damit sie ihren Studierenden Raum und Zeit geben, die Fremdsprache zu erlernen oder zu festigen. Bei „Fachsprachenunterricht“ ist der Bezug zum Fach unmittelbar einleuchtend, bei performativem Unterricht mit Gedichten, Theater oder Musik eher nicht. Dennoch profitieren Studierende in hohem Maße von einem holistischen Ansatz, der mehr als die kognitive Ebene umfasst und anspricht. Dieses ist beharrlich weiter zu kommunizieren, zu erklären und am besten, wo möglich, durch gesicherte Forschung und Beispiele zu 
belegen. Das Symposium in seiner Vielfältigkeit bildet performatives Lernen im Fremdsprachunterricht aber auch disziplinenübergreifend ab und kann so zum besseren Verständnis der Chancen und Möglichkeiten dieser Methode beitragen.

\section{Bibliografie}

Stöver-Blahak, Anke (2012): Sprechen und Vortragen lernen im Fremdsprachenunterricht. Interpretativ, kreativ und ganzheitlich mit Gedichten. Frankfurt a.M.: Peter Lang 